\title{
UN JOURNALISME DU COIN DU FEU
}

\author{
Denis Ruellan ${ }^{1}$ et Yvon Rochard ${ }^{2}$
}

La loi française de 1935 définissant le statut des journalistes a rendu illégitimes du point de vue professionnel les collaborateurs, notamment, en province, les correspondants locaux de presse (CLP). C'était même là un des objectifs principaux des promoteurs de cette loi.

Pourtant, dès l'instant du vote, les journalistes ont dû tenir compte du dualisme que générait la persistance de ces collaborations, nécessaires -bien que déconsidérées- à l'équilibre économique de la presse.

C'est dans cette contradiction que réside l'ambiguïté de la situation des correspondants de presse locale, qui n'est pas sans rapport avec la complexité générale du statut des gens de presse.

En effet, d'une manière générale, il existe un décalage important entre la catégorie considérée comme légitime à faire du journalisme et l'activité journalistique telle qu'elle est réalisée. Les journalistes légalement reconnus ne sont pas les seuls, loin s'en faut, à faire du journalisme, sous une forme ou une autre.

1 Professeur des universités, Université de Rennes 1 - IUT de Lannion.

2 Journaliste, Maître de conférences associé, Université de Rennes 1-IUT de Lannion. 
La presse a toujours utilisé des personnels qui ne peuvent entrer dans la catégorie légitimée par la loi actuelle : des collaborateurs occasionnels (intellectuels, voyageurs, critiques...) et d'autres, réguliers, mais pour qui le journalisme constitue une activité secondaire (c'est le cas des correspondants de presse locale). La loi a distingué la catégorie non pas par la définition de la nature du journalisme, mais par les caractères régulier et majoritaire du temps dévolu au journalisme. En général (mais les exception sont courantes), les correspondants de presse locale disposent d'un emploi principal (enseignants, fonctionnaires, techniciens de l'industrie, commerçants, retraités...), qui justifie leur exclusion du statut.

On ne sait pas aujourd'hui combien de personnes participent à l'activité journalistique (un travail intellectuel sur l'actualité), mais on conçoit que ce nombre égale celui des 36000 journalistes "encartés" (disposant de la "carte de presse"). La presse régionale est la plus grosse utilisatrice de cette force de travail : 65 quotidiens et 250 hebdomadaires emploieraient quelques 35000 correspondants de presse, tandis que les journalistes n'y sont que 6000 .

Alors que le journaliste est défini par une loi qui le protège et organise toute sa condition de salarié, gérant paritairement des pans importants de son univers professionnel ("carte de presse", commission arbitrale des conflits, convention collective...), le correspondant de presse, longtemps maintenu dans un flou juridique, est défini, depuis 1993, comme un travailleur indépendant, apportant une libre contribution à la presse, sans aucun lien de subordination. Les entreprises éditrices restent maîtres du droit de publier ou non des informations fournies et elles ne sont aucunement obligées vis-à-vis de ces contributaires.

En réalité, le correspondant de presse locale, pion de l'échiquier du marché de l'information de proximité, est, par sa multiplicité, une pièce maîtresse du jeu journalistique en région. On estime que de 40 à $60 \%$ de la surface rédactionnelle d'un grand quotidien, dont la force commerciale réside dans l'étroitesse de son maillage, dépend de la contribution des correspondants. Certains, ne se contentant pas de fournir de l'information avant mise en forme par les journalistes en titre, rédigent eux-mêmes articles et rubriques concernant leur aire géographique ou leur domaine de spécialité. En bien des cas, le correspondant remplit une fonction de nature journalistique, quand bien même il n'est pas reconnu comme journaliste. C'est dire son importance, pourtant totalement méconnue, voire cachée. 
C'est pour mieux percevoir cette dualité (source d'injustice, surtout en ces temps de chômage et de précarité qui favorisent les "petits boulots" et autres sous-emplois) que nous avons entrepris une enquête auprès de correspondants travaillant pour le compte de la presse régionale.

Nos interrogations portaient sur trois plans :

- l'activité concrète de ces correspondants ;

- la représentation de leur rôle et de leur statut en tant que travailleurs

(vis-à-vis des journalistes, de l'employeur et enfin de la société locale dans laquelle ils se situent);

- leur contribution à l'élaboration du journal ;

- leur contribution aux débats politiques et aux initiatives sociales locales.

Pour les besoins de l'étude, nous avons limité notre observation à un hebdomadaire local (le Trégor) disposant d'un réseau de 18 correspondants locaux. Les entretiens approfondis ont été menés avec 15 d'entre eux, entre mars et août 1996. Ces observations ont été complétées par des informations parcellaires issues de cinq grands quotidiens régionaux français (La Voix du Nord, Sud-Ouest, OuestFrance, La Dépêche du Midi, Le Télégramme).

Nous sommes parvenus à constater que les correspondants de presse locale tiennent une place essentielle non seulement dans leurs médias, mais aussi dans leur milieu de vie où ils assurent un travail de lien social, de transmission, de transparence, de valorisation, indispensable à la démocratie.

Notre contribution se propose de rapporter ces résultats en soulignant les interactions existant entre l'évolution d'un pays (au sens de micro région culturelle, ici le Trégor, sur la côte nord de la Bretagne), l'entreprise hebdomadaire d'un journal (le Trégor) et l'activité de ces observateurs-acteurs pratiquant un "journalisme du coin du feu", essentiel à l'entretien de la polémique locale.

\section{Un pays, un journal}

Le Trégor est le fruit d'une étonnante union entre deux hebdomadaires locaux, Le Lannionnais Républicain et L'Écho de Lannion. Le premier, héritier direct d'une feuille de chou créée en 1848 par un imprimeur, assumait, tout en s'adaptant à l'évolution des mœurs 
politiques, l'histoire républicaine et anticléricale de son aïeul. Le second, en filiation directe lui aussi avec un petit journal, né en 1833, défendait, avec la même fidélité, des valeurs opposées. En clair, l'un était rouge, l'autre blanc et, dans les années 1960, si les temps n'étaient plus à l'échange permanent d'invectives, les deux hebdomadaires affichaient encore, en période électorale, les clivages politiques ancestraux.

Autour d'eux, plus vite qu'eux, le monde se transformait: les mutations de l'agriculture, base de l'économie trégorroise; l'exode massif des jeunes actifs ; l'affaiblissement de l'identité culturelle... tout, à l"exception du tourisme, contribuait au déclin d'une région rurale dont Lannion, avec ses 6000 habitants, était la modeste capitale.

C'est dans ce contexte, qu'à la fin des années 1950; parvient de Paris l'annonce de la décentralisation en Bretagne, et plus précisément à Lannion, du Centre National d'Études des Télécommunications (CNET). Cette décision, largement influencée par l'origine trégorroise du directeur général du CNET, Pierre Marzin, et prise à une période ou les transmissions téléphoniques entament une phase de développement spectaculaire va transformer le pays de Lannion : la petite ville et les communes rurales voisines vont vivre une révolution économique et sociologique.

Les deux hebdomadaires sont pris dans un tourbillon qu'ils subissent dans un premier temps. Sollicités par les nouveaux acteurs du développement, ils investissent un peu plus le champ du social, de l'économie, du culturel. Leur contenu s'enrichit, leurs ventes augmentent mais, pour que leurs journaux soient à la hauteur du bouillonnement qui se produit, pour qu'ils puissent rendre compte de la mutation que vit la région, les deux imprimeurs-éditeurs comprennent qu'il leur faut se donner des moyens nouveaux et coûteux que chacun d'entre-eux ne peut envisager séparément. Deux hebdos, c'est un de trop : ils vont donc surmonter avec réalisme leurs divergences et s'unir pour créer, en 1970, un hebdomadaire qui prendra le nom du pays, pourtant tombé en désuétude, le Trégor.

Dès le premier numéro, en novembre 1970, avec 6500 exemplaires vendus, Le Trégor réunit les lectorats de ses deux prédécesseurs. Sa progression sera spectaculaire : 15000 en 1978; 20000 en $1990 ; 23000$ en 1995 -presque autant que les deux quotidiens OuestFrance et Le Télégramme réunis-, soit un taux de pénétration 
supérieur à $50 \%$ des foyers dans un peu plus de six cantons, une soixantaine de communes et environ 90000 habitants.

Pour parvenir à ce résultat exceptionnel, l'Impram, société éditrice du Trégor, a, au fil des années, considérablement investi dans des outils performants (groupes de rotatives et équipement informatique notamment) et dans des moyens humains. Une première journaliste est embauchée lors de la création, en 1970, ainsi que deux correspondants. Un second journaliste vient renforcer la rédaction en 1973 -année de l'acquisition de la première rotative-, puis un troisième en 1976, tandis que le réseau de correspondants s'étend progressivement et qu' augmente la pagination.

En quelques années, Le Trégor s'impose comme outil d'information locale mais aussi comme lieu de débat privilégié. Son enracinement, sa proximité, grâce notamment à son réseau de correspondants, sont renforcés par une souplesse dans la pagination, dans la maquette, dans les délais de bouclage qui lui permet de réduire le handicap de sa périodicité face aux quotidiens. Des quotidiens qui s'astreignent à la règle du "faire court", tandis qu'au Trégor règne celle du "faire plus", que les correspondants ne craignent pas les ciseaux du secrétaire de rédaction et que les citoyens occupent sans le moindre souci de l'économie les tribunes libres que le journal leur offre depuis sa création.

\section{Les correspondants, "infanterie" du Trégor}

En trente ans, le Trégor est passé des quatre pages du premier numéro à un volume moyen d'une soixantaine de pages (jusqu'à 72 parfois !) aujourd'hui. Cette croissance exponentielle que les évolutions technologiques et le succès commercial du journal ont rendu possible est le fruit d'une politique éditoriale qui a sensiblement évolué dans le sens notamment d'un accroissement de la place et du rôle occupés par les correspondants.

Après une courte période qui faisait du Trégor le digne héritier de ses deux parents en offrant chaque semaine une collection de communiqués locaux institutionnels précédés d'une série de nouvelles (brèves pour la plupart) régionales, nationales et internationales, une première étape a été franchie, en 1973, avec l'arrivée d'un journaliste formé à l'école de Lille. Outre une énergie débordante, celui-ci apportait un regard nouveau, une conception plus moderne du métier. Gildas Le Bozec était convaincu qu'un journal doit savoir se rendre 
utile, et même indispensable : il lui faut donc des antennes, des relais, des correspondants locaux qui transmettent en temps et en heure le compte rendu du conseil municipal, l'annonce du concours de boules ou de la fête des chasseurs. Le succès commercial de l'hebdo permet à l'entreprise d'embaucher ces collaborateurs au demeurant peu exigeants financièrement puisqu'ils sont secrétaires de mairie, instituteurs, retraités.

Le nouveau venu conçoit le journaliste comme un homme de terrain qui observe, écoute, s'immerge dans la société dont il parle et à laquelle il s'adresse. L'arrivée d'un troisième journaliste, en 1976, renforcera cette conception. Ils sont de cette génération qui pense que la qualité d'un journal se mesure à sa capacité à relayer, voire à animer les débats de société; à remettre en cause les pouvoirs et les conservatismes. Or, le pays du Trégor est en pleine mutation. Bons ou mauvais, les équilibres traditionnels sont ébranlés. La vie associative se régénère, se diversifie ; les conservatismes sont mis à mal. Autour de Lannion, comme par contagion, les hommes d'hier, maires' et conseillers municipaux, responsables d'associations, cèdent la place les uns après les autres à une nouvelle génération. Ce changement, signe sans doute de la forte identité de ce pays bretonnant, se produit sans drame, sans fracture, sans que le Trégor y perde son âme.

Cette nouvelle élite trégorroise, globalement en phase avec la politique éditoriale du Trégor participera largement à la réussite spectaculaire du journal dans la seconde moitié des années 1970. Mais en comparaison de cette pratique journalistique, exceptionnellement dynamique pour un hebdomadaire régional, la production des correspondants ne pouvait apparaître que décalée, un peu comme si deux Trégor coexistaient sous un même titre. Deux événements forts vont amener la rédaction à s'interroger sur ce déséquilibre et, partant, sur la place des correspondants locaux. Le premier, au printemps 78 , est l'arrivée très inquiétante de La Dépêche Trégoroise, un concurrent piloté par Le Télégramme qui avait auparavant échoué dans sa tentative pour racheter le Trégor-ce que fera Ouest-France en 1980.

La riposte de l'assiégé est de faire plus et de faire mieux, et, dans cette stratégie, les correspondants seront l'infanterie. Ils auront plus de moyens et plus de pages que la rédaction -elle-même prêchant d'exemple- les incitera à remplir avec un esprit de compétition accru. Cette stratégie nouvelle est renforcée, après 1980, par l'embauche de nouveaux correspondants qui viennent remplacer ceux qui, depuis l'achat de l'hebdo par Ouest-France, travaillaient pour les deux 
journaux au grand dam de nombreux lecteurs qui se sentaient floués, témoignant ainsi de l'importance qu'ils accordaient aux rubriques locales. A propos de cette période, Gildas Le Bozec, rédacteur en chef jusqu'en 1990, rapportait dans la revue $\operatorname{Ar}$ Men : "Nous avions aussi cherché une implantation populaire : si un concours de boules n'était pas annoncé dans le Trégor, il était annulé !' Cette implantation populaire, sans laquelle la courbe des ventes du journal aurait vite trouvé ses limites, dépendait très largement de la qualité du travail de veille des correspondants.

Le second événement qui amènera la rédaction du Trégor à réfléchir en profondeur sur sa politique éditoriale et sur le rôle de ses CLP est la stagnation des ventes enregistrée à partir de 1984. La crise de la téléphonie secoue alors Lannion et sa région, révélant la fragilité d'un développement basé sur une mono-industrie. Le Trégor est sur le front. Ses journalistes relatent en long et en large toutes les phases de la crise. Ils analysent, ils recueillent les témoignages, se font l'écho des craintes, des colères. Ils se démènent, noircissent les pages mais, pour la première fois, la courbe des ventes ne grimpe plus.

Dans ce climat de morosité, les responsables de l'hebdomadaire choisissent de valoriser plus encore l'information locale, de mieux jouer la carte de la proximité. C'est une tendance qui fait déjà son chemin dans la presse quotidienne régionale où l'on considère qu'une multitude de petites informations concernant la vie quotidienne et les préoccupations concrètes des gens valent mieux que de longs sujets aussi élaborés et bien écrits soient-ils.

La stratégie du Trégor s'oriente clairement, à cette époque, vers un renforcement des chroniques locales. Les conseils aux correspondants, écrits et oraux, vont tous dans le même sens : leurs rubriques doivent mieux refléter et rendre compte de la vie locale, de sa vigueur, de sa diversité, de son originalité.

La rédaction entreprend, à travers une série de documents maison, inspirés des cours de journalisme à l'usage des localiers, d'enrichir quantitativement et qualitativement la production des CLP. Ces derniers sont, par exemple, incités à rédiger des portraits :

Le portrait isolé : le receveur des postes est un œnologue expérimenté. La cantinière est une chanteuse émérite. Le fils du coiffeur est un musicien hors pair (...) La vie d'une association à travers l'action d'un homme (...) Françoise est une des membres actives du club de gym. Henri est la mémoire du club cyclo (...) Autant de sujets qui existent dans toutes les 
communes et qu'il est bon de soulever. Les infos de ce type sont encore trop rares dans certains cantons. Un effort a été fait, continuons-le. (...) Ces gens sont grands, petits, bruns ou blonds. Ils ont des choses à dire. Ils ont des commentaires à faire sur la vie locale. A travers leur regard sur l'actualité s'éclairera aussi celui du lecteur. Sans ces hommes et ces femmes qui font vivre les communes et la vie sociale, rien ne serait. C'est d'eux dont il faut parler. Sève de leur commune, ils le seront aussi pour le journal. Un portrait par semaine par canton serait une bonne vitesse de croisière. pensez-y!.

Autre stratégie rédactionnelle conseillée : l'encadré. Il s'agit de briser le rythme monotone des informations en relevant l'une d'elles et en lui apportant un développement particulier :

Les compte rendus traditionnels d'association ne constituent pas des encadrés en puissance. Par contre, la menace de destruction d'un immeuble ancien, la crise au sein du bureau du club du troisième âge, la fermeture du dernier commerce local, en sont. Il faut rebondir sur l'information et ne pas se laisser manger par le courant. (...) C'est aussi une façon de retrouver du dynamisme pour nous et le journal ${ }^{2}$.

\section{3. "La vie des plus humbles est un roman"}

En 1973, quand le Trégor franchit une étape importante de son développement en s'équipant d'une rotative, il n'emploie que deux correspondants. Treize ans plus tard, ils sont vingt à "seconder" les cinq journalistes de la rédaction. Leur contribution représente, approximativement et en moyenne, $60 \%$ du volume de copie publiée chaque semaine par le Trégor. Elle correspond à une bonne vingtaine de pages d'environ 10000 signes chacune, la part de chaque correspondant variant dans un rapport de 1 à 3 ou 4 .

La place consacrée à chaque commune varie considérablement en fonction de son importance, de sa vitalité et, bien entendu, des aléas de l'actualité. Cette dernière est traitée sous la forme de photoslégendes, de brèves de quelques lignes et d'articles, illustrés ou non, dont la longueur moyenne est de l'ordre de 50 lignes journal, soit un peu moins de 2000 signes. Les quelques rares textes qui approchent

\footnotetext{
1 Extrait d'un précis de conseils fourni par la rédaction.

2 Ibid.
} 
ou excèdent les cent lignes sont la plupart du temps des compte rendus de conseils municipaux traités sous une forme administrative et dans un corps inférieur.

En observant de près les articles produits par les correspondants, on constate, sans surprise, qu'ils sont essentiellement liés à l'agenda, c'est-à-dire aux événements programmés, aux temps forts de la vie des organismes qui structurent la communauté locale : réunions, manifestations, fêtes, etc. L'analyse de leur contenu est plus intéressante : elle révèle que le temps du "correspondant-boîte à lettres" est révolu, que son rôle ne se limite plus à collecter les communiqués fournis par les organisateurs de ces réunions et manifestations diverses puis à les transmettre au journal après une sommaire relecture.

Dans la majorité des cas, en effet, le correspondant effectue un travail à partir de l'information brute : parfois réduit à sa plus simple expression, parfois maladroit ou incomplet, mais incontestablement journalistique puisqu'on y trouve une recherche, une analyse et une sélection de 1'information. Cette recherche intègre très souvent un entretien avec une personne jouant un rôle central dans l'organisation de l'événement concerné et dont une sélection de propos est utilisée sous forme de citations. Si la pratique de l'entretien s'arrête dans la plupart des cas à ce niveau, elle n'en est pas moins présente à chaque page de l'hebdomadaire et participe de ce "travail intellectuel sur l'information" qui définit l'activité journalistique.

Dans un premier temps, laissant aux quotidiens régionaux le soin de traiter l'information régionale et nationale, le Trégor avait choisi de développer quantitativement l'information locale. Cette stratégie reposait sur la complémentarité journaliste-correspondants, mais aussi sur une distinction dans la définition de leurs missions. Aux seconds revenait l'approvisionnement du journal en informations brutes, non élaborées, tandis qu'il incombait aux premiers de creuser, de mettre en forme journalistique les sujets jugés importants, relatifs, la plupart du temps, à la ville-centre, Lannion, où à un ensemble plus vaste.

La seconde étape a consisté à valoriser qualitativement l'information collectée par les correspondants. Bernard Rubin, rédacteur en chef, écrivait ainsi, en 1990, dans le numéro consacré aux 20 ans du journal :

Nous avons laissé à d'autres le soin de raconter le monde et la France, pour nous attacher davantage aux moindres recoins de 
ce pays que nous aimons. Car la vie des plus humbles est un roman. Car le mariage d'un Trégorrois est aussi important, pour notre vie ici, que celui de la princesse de Monaco. Car la vie de l'équipe de foot de Langoat, de Trémel ou de Cavan, est aussi passionnante que celle de l'Olympique de Marseille.

Cette défense et illustration du journalisme de proximité fait du correspondant local un journaliste, un grand reporter dans les limites de sa zone d'action. Et si les journalistes professionnels de la rédaction du Trégor interviennent effectivement sur la copie des correspondants, leur intervention se situe dans des limites très comparables à celles des secrétaires de rédaction vis à vis de la copie des journalistes qu'ils ont pour mission de relire, de corriger, d'habiller souvent de titres et d'intertitres, et de modifier parfois en fonction d'une politique éditoriale ou des exigences techniques.

\section{Un compromis d'aspirations}

Le recours à la correspondance résulte d'un étroit compromis entre les intérêts du journal et les aspirations des correspondants.

De son côté, le journal trouve dans le système d'emploi des CLP le moyen de mettre en œuvre un maillage très fin de la collecte de l'information. En moyenne, chaque CLP couvre une population d'environ 4600 habitants, répartis sur une à neuf communes. Sa proximité des sources d'information, qu'il côtoie quotidiennement dans la vie courante, constitue un instrument de veille permanente très efficace. Aucun événement susceptible d'intéresser les lecteurs ne risque d'échapper à la vigilance du journal. Si la lecture par un regard externe des pages locales donne l'impression d'un remplissage de colonnes par des faits à la portée insignifiante, cette appréciation n'est pas partagée par les lecteurs des localités qui, au contraire, trouvent dans ce foisonnement la satisfaction de leur besoin d'information. Contrairement à la culture journalistique qui conçoit que la relation de l'actualité doit être brève et concise, le journal local trouve dans la longueur et le détail le moyen de répondre aux attentes des lecteurs. Ceux-ci, évidemment, ne lisent pas l'intégralité des pages locales, mais avant tout celles qui concernent leur localité et celles où ils peuvent être conduits à travailler ou avoir des activités sociales.

Pour effectuer cette relation quasi ethnographique du quotidien, le CLP, membre de la localité, est le plus à même ; un journaliste 
professionnel ne saurait mieux remplir cette tâche; sans doute serait-il moins bien armé s'il devait, proportionnellement à son statut de travailleur permanent, couvrir une population et un nombre de communes quatre fois plus important (en considérant qu'un CLP consacre en moyenne un quart de son temps à la correspondance locale). On mesure ainsi l'intérêt économique du système : le CLP coûte très peu cher au journal par rapport à un salarié (trois à quatre fois moins). Mieux, il occasionne un coût de revient d'autant plus faible qu'il rend un service qu'un journaliste ne pourrait effectuer aussi efficacement car, si ses conditions de travail sont très souples, il offre, en contrepartie, une disponibilité sept jours sur sept et des horaires élastiques (dimanche, fériés, soirées, contrainte téléphonique...). Le journal cherche encore à optimiser cette rentabilité en incitant les CLP à s'équiper de moyens informatiques permettant d'éviter la saisie au journal.

De leur côté, les CLP tirent profit de cette activité sous rémunérée et mal reconnue professionnellement. Il n'est pas indifférent de dire que ces personnes sont ouvertes et curieuses. Leur niveau d'études important, leurs origines sociales relativement élevées, leur niveau d'emploi professionnel leur donnent les moyens d'appréhender la diversité et la complexité des situations sociales. L'expérience personnelle contribue beaucoup aussi à cette ouverture.

Les correspondants locaux (CLP) expliquent qu'ils exercent cette curiosité comme une contribution à la société à laquelle ils veulent appartenir pleinement. Ils ne sont pas en déficit d'insertion, au contraire : la quasi-totalité dispose d'un emploi principal stable ou d'une situation sans ambiguiité (retraite, mère au foyer) ; en principe, il n'y a pas de demandeurs d'emploi car le Trégor ne souhaite pas être à la merci des changements de situation professionnelle de ses correspondants.

Les CLP assument donc leur fonction comme un service à la collectivité : ils veulent valoriser le local, les initiatives et les personnes avec lesquelles elles vivent. Ils ne souhaitent pas, dans leur grande majorité, être les gendarmes de la vie sociale, mais son miroir -au risque de la complaisance.

Néanmoins, ils évitent d'être impliqué directement dans la vie locale et ils l'expliquent par la nécessité de conserver une distance vis-à-vis des sources. Le manque de temps est aussi une raison sérieuse. On peut y voir également une attitude individuelle commune : les CLP apprécient cette manière un peu à l'écart, plus 
solitaire, peut-être surplombante et privilégiée, d'exprimer leur esprit solidaire à l'égard d'une collectivité dont ils espèrent une reconnaissance (qui ne leur est pas refusée, bien au contraire). Ils se veulent des observateurs au service d'une collectivité, sans devoir s'impliquer plus.

La volonté de travailler dans une entreprise de presse est forte. Les CLP aiment être associés à cette entreprise collective de fabrication d'un journal. Les relations avec la rédaction demeurent insatisfaisantes pour nombre d'entre eux ; on souffre du manque de contacts dû à l'éparpillement dans les communes et le fait que chaque travaille chez lui, le siège du journal n'étant fréquenté au mieux que quelques minutes par semaine ; on se sent aussi à part des journalistes statutaires, on ressent un déficit de reconnaissance. Néanmoins, les CLP apprécient l'indépendance de fonctionnement vis-à-vis de l'employeur. En principe (en raison du statut de travailleurs indépendants), ils ne reçoivent pas d'ordres ; éventuellement on leur communique des instructions et des conseils (mais jamais aucune formation, celle-ci se faisant "sur le tas", du jour pour le lendemain car la presse ne s'arrête jamais) ; ils choisissent eux-mêmes les sujets et la manière de les traiter.

L'aspect financier n'est pas à négliger. Le faible rapport de rentabilité est compensé par la souplesse du système. Pour tous les CLP, ce revenu est important, même s'il se fait moins indispensable pour certains. Et, de plus en plus, il semble associé à un travail de complément, un petit boulot permettant de financer les dépenses supplémentaires (loisirs, études...).

Le CLP est un personnage interface. Pour l'entreprise, son rôle est à double détente : il recueille de l'information, et cette information va justifier l'achat par les lecteurs. L'absence de CLP dans une commune, ou la déficience de son activité, entraîne naturellement un infléchissement des ventes dans la localité concernée. Avoir un bon CLP est, pour le journal, le gage d'une veille efficace et de ventes en rapport. De ce point de vue, le CLP est le représentant du journal dans sa commune. Même s'il n'est pas chargé de la distribution, il en est un agent essentiel. Par ailleurs, le CLP est le représentant du local dans le journal. Grâce à lui, les groupes et individus qui forment les sociétés locales sont assurés de la qualité d'un service d'information nécessaire à la vie sociale et économique. Les CLP témoignent tous de la très bonne réception de leur activité ; ils se disent intégrés et acceptés, sans doute mieux que des journalistes professionnels. Cela provient 
du fait qu'ils répondent aux attentes locales : on espère d'eux qu'ils transmettent l'information et qu'ils valorisent ainsi la vie locale à travers un outil dont tout le monde à besoin. Autrement dit, ils défendent les intérêts de leur collectivité au sein d'un instrument commun à une région.

Le CLP tient un rôle bien précis dans la répartition des tâches journalistiques : il assume une information de service, dénuée de conflits - et parfois même de débats-, laissant aux journalistes le soin d'assurer le traitement des sujets à enjeu fort. Cette répartition souligne que le journalisme n'est pas, comme on a tendance à le croire, qu'une activité de révélation du conflit social. La tradition de la presse héritée du XVIIIe siècle, tend à ne considérer que l'information à dimension politique. Le journalisme du local (assumé par les correspondants principalement) rappelle avec force que l'information est aussi un élément du simple lien social : les êtres qui vivent en collectivité ont besoin d'une somme variable d'informations pour se situer et développer leur activité ; ils ont aussi besoin d'un instrument de transparence de la vie politique. Correspondants et journalistes se répartissent donc, au mieux, la tâche : de par leur proximité, les CLP sont mieux placés pour assumer l'information de service; de par leur distance, les journalistes peuvent traiter les sujets plus conflictuels.

Cette répartition peut souffrir d'exception : à la demande de certains CLP (qui veulent prolonger leur tâche journalistique vers des sujets plus complexes) ou à celle du journal (qui apprécie d'être épaulé dans sa tâche de relation des enjeux locaux), des CLP en viennent à confondre leur rôle avec celui des journalistes ; cette dérive est alors porteuse de frustrations et de mésententes. Cette tendance est sans doute plus préoccupante qu'il n'y paraît. En effet, la qualification élevée des CLP d'une part, l'introduction de technologies nouvelles d'autre part (de plus en plus de CLP s'équipent de moyens informatiques performants, certains assument même la mise en page et le temps n'est pas loin où ils transmettront leur copie par réseau téléphonique), conduisent à la confusion des rôles. Par son attitude, le journal semble d'ailleurs aller dans ce sens, porteur de conflits à moyen terme. 


\section{Conclusion}

Si le système a d'indéniables avantages, il tend à maintenir le journalisme du local dans un carcan qualitatif (que la confusion des rôles semble vouloir faire exploser, signe de la difficulté). En effet, maintenus à la marge du journalisme, non formés, mal payés, les CLP "tirent à la ligne" plus qu'ils ne font du journalisme : la relation qu'il font du local souffre de distance, de vigueur, d'originalité ; c'est une œuvre répétitive, satisfaisante mais certainement perfectible. Le profil bas qu'adoptent la plupart, très compréhensible dans les conditions actuelles, interdit une vraie valorisation des initiatives et des richesses du local. Quelques correspondants tentent d'extraire leur fonction de la routine : ils cherchent de l'information au delà de celle qu'on leur apporte ; ils enquêtent, fouillent et dénichent des aspects inconnus. C'est là une démarche encore personnelle, qui trouve mal sa place dans le système actuel.

Le journal en a conscience et essaye d'en tirer parti, en incitant les volontaires à continuer dans cette voie. Il attire aussi l'attention de tous les CLP sur les nouvelles manières de traiter l'information. Un premier pas avait été franchi avec l'introduction de la photographie dans les pages locales et la généralisation des photos-légendes, ces informations exprimées par une photographie très brièvement commentée. Une autre étape fut la multiplication des brèves qui permit d'étendre la veille aux informations courantes (état-civil, permis de construire, déménagements, départs en retraite, nominations, gains au loto, licenciements, expansion des entreprises...) et de dépasser l'information institutionnelle (municipalités, associations). Désormais, c'est sur les sujets plus fouillés que le Trégor met l'accent, notamment en valorisant financièrement leur traitement. Tous les correspondants ne semblent pas prêts à cette mutation, qui implique une démarche journalistique plus fouillée, plus entreprenante. Mais le Trégor croit à cette évolution, au risque de confondre plus profondément un jeu de rôle savamment établi.

Il demeure que le système de la correspondance, placé en marge du journalisme statutaire, est une remarquable machine à renouveler le journalisme en son cœur. Cette forme d'information d'hyperproximité, intimement liée à la vie publique et privée des villes et villages, ce journalisme du coin du feu n'existe que par les correspon- 
dants locaux. De par leur réalité structurelle (ils sont affectivement et physiquement plus lointains) et de par leur culture professionnelle (essentiellement inspirée par le journalisme politique parisien, faisant de l'indépendance la vertu cardinale), les journalistes statutaires ne sont pas prêts à assumer ce niveau laborieux. Or, c'est bien là que l'information locale moderne se définit, où elle est indispensable et fait la fortune des entreprises de presse. Le succès de la correspondance oblige à repenser le journalisme, dans ses finalités et dans ses organisations. 